\title{
STRUCTURES ET FONCTIONNEMENT SÉMIOTIQUES DES ICONES DE LOGICIELS ET D'ENVIRONNEMENTS INFORMATIQUES STANDARDISÉS (ILEIS)
}

\author{
Daniel Peraya ${ }^{1}$
}

\section{En guise d'introduction : une lecture de quelques icones}

\section{Un fidèle ami}

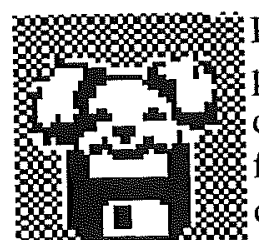

Parmi les icones qui peuplent les logiciels standards, prenons celle-ci qui provient de l'univers Mac : elle désigne le logiciel Fetch qui permet les transferts de fichiers entre des machines distantes selon le protocole de transfert de fichiers FTP. On y reconnaîtra sans trop de difficulté la représentation analogique d'un animal tenant entre les mâchoires une disquette : elle se compose donc de deux éléments simples, la disquette et l'animal. Mais est-ce un chien, éventuellement un koala ou un autre animal ? Lorsque l'on

1 Maitre d'enseignement et de recherche, TECFA, Unité des technologies éducatives, Faculté de Psychologie et des Sciences de l'Éducation, Université de Genève.

Recherches en communication, $\mathrm{n}^{\circ} 10,(1998)$. 
interroge des utilisateurs, le koala est souvent cité surtout face à la version plus récente, en couleurs. Enfin, quelques rares sujets nous ont proposé la souris. Dans notre contexte culturel, l'interprétation penchera le plus souvent pour le chien et la compréhension littérale de cette icone, sa dénotation, s'imposera en partie à cause de la métaphore qui lui assure sa cohérence sémantique.

Le "chien" me regarde et semble même me sourire : sa gueule - un arc de cercle ouvert vers le haut - rappelle le signe analogique du sourire, ce tracé mimétique du mouvement de mes propres lèvres lorsque je souris. Cette représentation, ce mouvement intériorisé, s'est d'ailleurs figé et est depuis longtemps reproduite telle quelle dans de nombreux environnements graphiques (bande dessinée, logogrammes, smilies). Il y a là une part de convention dans un signe analogique. Quelle que soit la nature du lien sémiotique de cette gueule - disons de l'analogique conventionalisé -, je dois de toute évidence admettre qu'elle constitue une sous-entité apportant sa part à la construction du sens. Et si l'on peut difficilement dire qu'un chien sourit - encore qu'un certain anthropomorphisme n'est guère surprenant à propos de notre si fidèle ami ...-, tout au moins apparaît-il manifester à mon égard une certaine empathie. Ses yeux rieurs et ses oreilles exprimeraient d'ailleurs le même sentiment de contentement.

La disquette demande aussi quelques commentaires. Elle est à l'origine d'un mécanisme métonymique assez classique puisqu'elle représente non pas la disquette elle-même mais bien l'information, donc le(s) fichier(s) : elle désigne le contenu par le contenant et éventuellement, la partie pour le tout. Pourtant la métonymie n'est possible que si l'on accepte que la disquette représente d'abord tout support de stockage numérique (le particulier pour le général) et enfin, l'action de sauver par le support de stockage. On aurait donc au moins deux - sans doute trois - processus métonymiques enchâssés :

1) le support de stockage pour l'action de stocker ;

2) un support de stockage particulier pour tout support de stockage ;

3) le support de stockage pour l'information stockée.

Cependant ni les détails ni ces processus ne m'apparaissent lors de ma première lecture. Mes premières impressions nées d'une compréhension immédiate, globale, m'orientent vers d'autres significations. Cette représentation est conforme à l'image et à l'expérience commune - directe ou indirecte - que je puis avoir du chien : dans la rue il porte mes journaux, mais surtout il me rapporte les objets les 
plus divers : le bâton ou la balle que je lui lance, voire mes pantoufles quand je suis dans mon fauteuil à lire mon journal. Fetch déplace mes fichiers et m'amène ceux dont $\mathrm{j}$ ' ai besoin comme mon chien $m$ 'apporterait ces objets. A l'image de l'animal servile s'ajoute celle d'une amitié et d'un soutien indéfectibles, l'une et l'autre rejaillissant sur le logiciel qui revêt ces qualités canines fondamentales : collaboration, fiabilité, fidélité, etc. Le logiciel Fetch est pour moi ce chien fidèle et efficace : l'icone du logiciel fonctionne donc comme une parfaite métaphore.

Pour que la métaphore fonctionne, il faut encore que le chien me fasse face, qu'il soit en quelque sorte mon interlocuteur: qu'il s'agisse d'un ballon ou d'une disquette, c'est à moi qu'il les rapporte, les présente et les offre. D'ailleurs, la collaboration et la fidélité impliquent naturellement le partenariat. La structure formelle de la représentation et la mise en scène des éléments figurés - la posture du chien, son regard qui me regarde et son "sourire" -, m' assignent une place et un rôle dans le dispositif d'énonciation ainsi créé. L'axe de ces regards croisés constitue une marque d'énonciation et m'introduit au registre du discours. La signification ne se déploie qu'à cette seule condition.

\section{Une certaine imprimante}

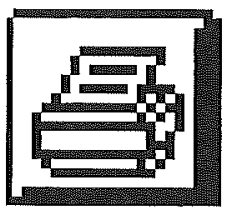

Prenons un deuxième exemple. Cette représentation d'imprimante est partagée par de nombreux logiciels et par plusieurs systèmes d'exploitation différents : elle orne le bouton qui commande la fonction d'impression du document actif. Elle connaît cependant des variantes: le logiciel Netscape présente une imprimante dessinée de face, sans perspective cavalière.

D'emblée, cette représentation paraît plus simple que celle du logiciel Fetch : il s'agit d'une imprimante. Malgré cela, elle met en œuvre différents processus sémiotiques. On s'aperçoit en effet qu'elle suppose une double métonymie puisque l'action d'imprimer est représentée et par l'agent de cette action - la machine - et par son résultat - la feuille imprimée.

Une analyse plus approfondie montre qu'elle met en ouvre deux registres sémiotiques : l'analogique domine, mais il existe aussi une part d'analogique conventionnel puisque la page imprimée est recon- 
naissable aux lignes d'écriture, représentées par des traits centrés continus. Elle est constituée de deux éléments principaux, de deux entités - l'imprimante et la feuille imprimée -, mais aussi d'une sousentité indispensable, les deux lignes d'écriture, qui donnent à la page sa signification propre. Enfin, cette représentation me semble objective et je ne me sens en rien impliqué par elle : elle ne présente aucune marque d'énonciation (note ${ }^{1}$ pour un jugement plus nuancé) et semble dire "ceci est un imprimante, voici une imprimante". Le dispositif d'énonciation semble conçu à la troisième personne, c'est-à-dire à la non-personne : contrairement au cas précédent, nous serions dans le registre du récit.

\section{Du texte}

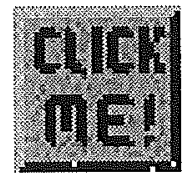

Regardons enfin ce bouton. Il présente un simple texte: "Click me!", une injonction en langue anglaise, composée de signes linguistiques, donc arbitraires et conventionnels. Peut-on encore parler d'icone puisque le registre sémiotique utilisé est strictement symbolique ? On répondra que oui car il s'agit de langage écrit et qu'en conséquence la représentation est de type scriptovisuel. Dans ce cas précis pourtant la mise en page et le traitement visuospatial sont peu exploités et réduits à leur degré zéro. En comparaison avec ceux des deux premiers exemples, les mécanismes de semiosis sont ici extraordinairement simples : l'énoncé linguistique suffit à la production du sens et l'impératif suffit comme marque d'énonciation.

Ces icones constituent trois exemples de fonctionnement sémiopragmatique différents. Elles mettent en œuvre des mécanismes caractéristiques : registres discursifs de l'ordre du récit ou du discours, registres sémiotiques et nature des représentations (indiciel, iconique et/ou verbal), exploitation de procédés connotatifs et associatifs ou simplement dénotatifs, structuration visuospatiale de l'information (rapport avant-plan/arrière plan, utilisation ou non de la perspective), degré de complexité, structuration entre entité et sousentités, etc.

Cette diversité et la richesse des procédés observés de façon encore fort intuitive devrait nous convaincre de la pertinence qu'il y

1 On pourrait encore argumenter sur le rôle de la perspective qui place l'œil du lecteur au centre du spectacle et de la représentation. 
aurait à systématiser l'approche des ILEIS et à dégager leur grammaire de base. Cette analyse devrait pouvoir contribuer à l'élaboration de réponses à de nombreuses questions de recherche concernant les registres sémiotiques et les représentations iconiques.

\section{Définir une problématique}

\section{Un phénomène en plein essor}

Les littératures pédagogique et technique ${ }^{1}$ utilisent depuis longtemps des petites plages visuelles standardisées - des icones afin de marquer et de structurer l'information. Les exemples sont nombreux et souvent anciens. Rappelons les guides touristiques ou culinaires qui proposent un véritable lexique d'icones pour caractériser les sites touristiques, les auberges, les relais et les hôtels. Dans les années ' 70 , les manuels de français de Mitterand et Mitterand publiés aux Nouvelles éditions africaines utilisaient déjà ce procédé pour indiquer à l'élève les paragraphes importants. Aujourd'hui, de nombreux cours imprimés de services de formation à distance (par exemple ceux de la Communauté française de Belgique ou la FernUniversität allemande) recourent à ce même procédé : les icones indiquent au lecteur le statut du texte et les tâches qui lui sont associées. Quant aux livres d'informatique, quels que soient la collection et l'éditeur, ils regorgent d'icones de ce type.

Très nombreuses encore dans les publications électroniques, les icones constituent de réels outils de structuration et d'ergonomie textuelles : elles font partie de ce que $\mathrm{Caro}^{2}$ a appelé récemment des organisateurs paralinguistiques. Elles signalent aux lecteurs la hiérarchie et l'importance des unités textuelles ou bien encore leur nature. Quant aux dispositifs de communication médiatisée par ordinateur - courrier électronique, plate-forme de communication et de collaboration -, ils constituent un champ d'expérimentation privilégié dans ce

1 La littérature enfantine ne semble d'ailleurs pas échapper à ce qui pourrait bien n'être qu'un effet de mode dans la littérature imprimée : dans une version illustrée récente du Petit Chaperon Rouge (Petit chaperon rouge (Le), Paris, Éd. Épigones, 1996). On relira aussi avec intérêt Dandan au pays du Berkberque, feuilleton dessiné des années 20.

2 S. CARO, Rôle des organisateurs paralinguistiques dans la consultation des documents électroniques, Thèse de Doctorat Sciences de l'Information et de la Communication, Grenoble, Université Stendhal, Grenoble III, 1995. 
domaine. On observe une tendance à marquer chaque intervention afin de signaler aux interlocuteurs la nature de l'acte de langage produit, d'expliciter ainsi l'intention de communication de l'émetteur et/ou d'exprimer un commentaire d'ordre métacommunicationnel. L'introduction de marqueurs d'affectivité - les smilies ${ }^{1}$ - montre bien la nécessité de pallier la nature "froide" selon l'expression de Mac Luhan $^{2}$ de la communication électronique. Ces marqueurs, préalablement définis, facilitent la catégorisation de chaque intervention et en conséquence contribuent à la régulation de l'interaction ${ }^{3}$. Dans cette perspective, les ouvreurs de discours et les modalités de réponse semistructurées font l'objet d'une grande attention de la part des chercheurs ${ }^{4}$.

\section{Un observatoire privilégié des processus de genèse des représentations iconiques}

Les ILEIS possèdent un triple statut, communicationnel, sémiotique et cognitif et ces trois aspects profondément liés sont constitutifs de ce type de production. L'hypothèse générale qui en sous-tend l'usage est qu'un marquage visuel constitue une aide efficace à la mémorisation et à la recherche de l'information. Selon le contexte d'utilisation et l'environnement de travail, cette aide peut prendre deux formes différentes. En premier lieu, dans le contexte de la publi-

1 Voir par exemple, The Smiley Dictionnary. Cool Things to do with your Keyboard, Berkeley, Peachpit Press, 1995.

2 M. MC Luhan, Pour comprendre les médias, Paris, Mame/Seuil, 1968 (pour la traduction française ; 1964, pour la version originale).

3 Voir par exemple l'usage qui en a été fait dans le projet JITOL (programme communautaire européen DELTA, phase II, 1992-1994), coordonné par l'Unité de traitement et d'enseignement pour diabétiques (UTED, Hôpital cantonal de Genève). L'objectif du projet consistait à mettre en réseau des membres d'une communauté scientifique et/ou professionnelle et de développer entre eux des interactions aux fins d'éducation et de formation. Le processus de traitement et de systématisation de l'information échangée - la "réification" - permettait une évolution constante du savoir disponible et permettait la constitution de banques de donnés dynamiques et évolutives. Le "marquage" de chaque intervention avait pour but de faciliter le processus de réification.

$4 \mathrm{M}$. BAKER, "A model for tutorial dialogues based on critical argument", in D. BIERMAN, J. BRUEKER, J. SANDBERG (Eds), Artificiel Intelligence and Education, 2-8, Amsterdam, IOS Publishers, 1989. Voir aussi P. JERMANN, Conception et analyse d'une interface semi-structurée dédiée à la co-résolution de problème, Mémoire de DES - STAF, Genève, TECFA, Faculté de Psychologie et des Sciences de l'Éducation, 1996. 
cation électronique, le lecteur aurait une meilleure représentation de la place et de l'importance relatives de chaque unité textuelle dans l'ensemble du texte. En conséquence, grâce à cette forme de mémoire externe, le lecteur pourrait mieux s'orienter dans le document, identifier, sélectionner et extraire l'information pertinente ${ }^{1}$.

Deuxièmement, dans les logiciels, les ILEIS seraient un moyen mnémotechnique pour accéder aux logiciels ou à leurs fonctions comme l'indique d'ailleurs le manuel en ligne de Visual Basic, le langage de programmation des interfaces graphiques sous Windows. On peut y lire par exemple cette définition: "icons are pictorial reminders of computers functions". En conséquence, les ILEIS concernent directement les processus cognitifs de mémorisation et elle sont des représentations de fonctions ou d'actions - imprimer, sauver, trier, remplacer, corriger l'orthographe, etc. - , au sens large. Dans ce cas d'ailleurs, l'icone est accompagnée d'un texte décrivant sa fonction, "ancrant" sa signification (voir note ${ }^{2}$ pour la définition de cette notion). Elle fait donc partie d'une stratégie plurimédia ${ }^{3}$, au sens où langage analogique et verbal se complètent et s'éclairent mutuellement.

Troisièmement, à cause du développement considérable d'Internet et d'outils logiciels, beaucoup de développeurs individuels créent, dans leurs propres produits - logiciels et sites Web-, de nombreuses icones (logo, icones de marquage, de navigation et de fonction, etc.) plus ou moins inspirées des ILEIS ou de catalogues spécialisés d'icones et de dessins. Rappelons qu'il existe aujourd'hui de nombreux logiciels spécialisés uniquement consacrés à la conception d'icones et de boutons graphiques. On observe donc une importante inventivité dans ce domaine qui pourrait être un terrain privilégié d'observation des pratiques de création et de stabilisation du langage des icones. Pour s'imposer comme élément d'un système,

1 Une hypothèse semblable expliquait déjà la fonction métatextuelle de certaines plages visuelles - de certains paratextes - dans la littérature imprimée et plus généralement dans les manuels scolaires. Pour une synthèse de cette question, voir D. PERAYA, M.-C. NYSSEN, Pour une théorie des paratextes : une étude comparée des manuels de biologie et d'économie, Les cahiers de la Section des Sciences de l'Education, $\mathrm{n}^{\circ} 78$, Genève, Faculté de Psychologie et des Sciences de l'Education, 1995, pp. 27-40.

2 R. BARTHES (1964), analysant à propos d'une publicité pour les pâtes Panzani, les rapports entre le message iconique et message linguistique attribuait à ce dernier une fonction d'ancrage.

3 Voir B. DARrAs, Au commencement était l'image, du dessin d'enfant à la communication d'adultes, Paris, ESF, 1996. Notamment pp. 25 et suiv. 
ces productions d'origine individuelle doivent faire l'objet d'un processus consensuel visant à négocier et à stabiliser leur "prétention désignative" à la fin duquel elles peuvent être partagées et, avec elles, leur signification. Il s'agit donc d'une véritable dialectique entre l'individuel et le collectif qui n'est pas sans rappeler celle entre la parole et la langue.

Enfin, à l'exception de quelques rares recherches comme celles de Guastello et al. ${ }^{1}$, la thématique a encore été relativement peu étudiée. D'ailleurs, l'étude mentionnée porte essentiellement sur l'effet de représentations mêlant le verbal' et l'iconique et sur la répartition du traitement de l'information entre les deux hémisphères cérébraux. Si elle semble apporter une confirmation à la théorie du double codage de Paivio ${ }^{2}$ et à la nécessité d'une forte interconnexion entre les informations verbales et iconiques ${ }^{3}$, elle demeure néanmoins fort éloignée du cadre de référence sémiopragmatique.

\section{Explorer les principaux thèmes et axes de recherche}

D'après ce que nous venons d'évoquer très brièvement, le lecteur comprendra que l'analyse approfondie de ILEIS permet de définir une problématique féconde ainsi que les principales questions recherche qui en circonscrivent les frontières.

\section{Représentations mentales et matérielles}

Les ILEIS sont des signes résultant d'un processus d'élaboration sémiotique de type onomasiologique. Cependant leur taille réduite constitue une contrainte déterminant une extrême simplicité de la représentation : elles sont constituées de peu d'éléments et ceux-ci sont le plus souvent de nature prototypique ; elles constituent donc un terrain idéal pour tenter d'articuler les théories des représentations

1 S. J. Guastello, M. TRAUT, G. KorIENEK, "Verbal versus pictorial representations of objects in a human-computer interface", International Journal of man-machine studies, 31, pp. 99-120, 1989. Cité par A. CLARKE, The principles of screen design for computer based learning material, Learning methods Project Report, OL121, Employment Department, Crown, 1989/1992.

2 A. PAIvio, Mental representation: A dual coding approach, New York, Oxford University Press.

3 J. R. KIRBY, "Collaborative and competitive effects of verbal and spatial processes", in Learning and Instruction, Comprehension of graphics in texts, 3, 3, 1993, pp. 201-215. 
matérielles et mentales, pour penser les rapports entre processus sémiotiques et cognitifs. Autrement dit pour jeter les fondement théoriques d'une sémiotique cognitive.

Le processus de création des ILEIS a-t-il, par exemple, un quelconque rapport avec la morphogénèse des iconotypes décrite par B. Darras ? Le cas échéant, il faudrait interroger les ressemblances mais aussi les dissemblances tant structurelles que fonctionnelles entre ILEIS et les iconotypes. Si, comme le soutient cet auteur, les pictogrammes - parmi lesquels on doit compter les icones de logiciels - sont des iconotypes stabilisés répondant à l'intention d'universalité, on devrait aussi, dans la perspective d'une généalogie des images, identifier les caractéristiques qui assurent le passage de l'iconotype au pictogramme.

\section{De l'iconique au verbal}

Les concepteurs de logiciels font afficher aujourd'hui dans une bulle d'aide la signification de l'icone. On peut supposer que la diversification et la nature des fonctions à représenter sont devenues trop complexes pour que la représentation analogique puisse seule supporter la signification monosémique que les icones sont supposées véhiculer. Le langage verbal et sa fonction d'ancrage s'imposeraient donc une fois de plus comme la clé de l'intelligibilité de l'image.

Comment les utilisateurs accèdent-ils à la signification de l'icone? Par un travail de décodage et de reconstruction du sens impliquant souvent de coûteuses inférences ou utilisent-ils le message linguistique ? Le cas échéant, quelles sont les interrelations entre les deux messages ? La convergence des registres sémiotiques - de nature iconique et verbale - étudiée par Duval ${ }^{1}$ joue-t-elle un rôle dans la mémorisation et dans l'accès aux diverses fonctions des logiciels ? On pourrait aussi se demander s'il existe une convergence entre d'une part les organisations topologiques et syntaxiques des ILEIS et d'autre part les structures linguistiques, syntaxiques, des énoncés verbaux qui les décrivent.

1 R. Duval, Semiosis et pensée humaine, Berne, Peter Lang, 1995. 


\section{La naissance d'une grammaire et d'une rhétorique}

Les ILEIS sont des représentations matérielles : existe-t-il des structures sémiotiques (notamment visuospatiales et topologiques) stables qui fondent leur construction ou leur " grammaire" ? Le cas échéant, quelles sont-elles ? Existe-t-il un rapport entre ces structures visuospatiales, ces catégories topologiques et celles d'autres formes de représentation graphique, telles que les iconotypes définis par B. Darras ${ }^{1}$ ? De plus, de nombreuses icones sont construites sur la base de procédés rhétoriques, métaphore et métonymie. Elles constituent donc un objet privilégié pour analyser les processus associatifs, rhétoriques et cognitifs qui sont liés à l'utilisation de tels procédés discursifs.

Étant donné le nombre important de créations individuelles, notamment dans le réseau et dans le WEB, on peut se demander encore quel est le rapport entre ces créations individuelles et le langage d'icones en voie de standardisation et de normalisation en partie grâce à l'énorme diffusion des logiciels standards. Autrement dit, il semblerait que l'on soit encore à une époque suffisamment créative pour observer la dynamique classique entre langue et parole, mais aussi le processus consensuel destiné à valider ces productions.

\section{Le point du vue cognitif}

On peut se demander enfin jusqu'à quel point la nature en grande partie analogique et métaphorique de ces représentations constitue une aide à leur interprétation et à la compréhension des tâches qui leur sont associées. Autrement dit, on pourrait tenter de définir la limite d'efficacité, selon les tâches à effectuer, des représentations analogiques. A partir d'une telle limite les représentations symboliques et conventionnelles deviendraient indispensables. Cette interrogation repose sur la spécificité de registres sémiotiques et sur leur capacité à traiter seulement certaines formes d'information ${ }^{2}$.

B. DarRas, op. cit.

2 R. DUVAL, op. cit. 


\section{Au-delà des enjeux théoriques}

Ce premier inventaire n'est bien sûr pas exhaustif; il n'a de valeur qu'indicative. Pourtant, donner une réponse à ces questions serait d'une extrême importance. Tout d'abord, ce serait une façon de contribuer au développement de la théorie sémiotique elle-même et à son avancée théorique. L'évolution du domaine dépend en effet de la réponse à certaines de ces questions fondamentales qui toutes portent sur l'intégration par la sémiotique des acquis du cognitivisme: l'articulation entre noésis et sémiosis, entre représentations mentales et sémiotiques ; la convergence et la structuration de registres sémiotiques différents', etc.

Mais les enjeux ne sont pas exclusivement théoriques. Une réponse claire à certaines de ces interrogations permettrait de résoudre de nombreux problèmes d'ergonomie et de design : lisibilité et intelligibilité des icones, choix entre des registres de représentation différents, degré de convergence entre l'icone et sa description linguistique, effet de seuil de la représentation analogique et de la métaphore, etc. La principale question de concepteurs, comme le rappelle Ben Schneiderman est en effet celle-ci : "How to decide between text and icon, and how to design icons"2. Enfin, dans une perspective pédagogique, on pourrait enfin se demander si une méthode rigoureuse d'analyse des structures des ILEIS et si une stricte méthodologie de production peuvent aider des non-professionnels (non-graphistes) et des professionnels dans la réalisation d'icones plus compréhensibles, plus fonctionnelles et plus adaptées aux utilisateurs.

\section{Un cadre d'analyse descriptive des ILEIS}

Le cadre descriptif que nous présentons ici se fonde sur plusieurs travaux antérieurs dont certains sont encore en cours aujourd'hui. Il n'est certes pas définitif et devra sans aucun doute faire l'objet d'une validation expérimentale ${ }^{3}$.

1 lbid.

2 B. SCHNEIDERMAN, Designing the User Interface. Stratégies for effective HumanComputer Interaction, New York, Addison-Wesley Publishing Company,1992, pp. 207 et suiv.

3 Cette recherche est née il y a trois ans dans le cadre du diplôme STAF de l'Unité des technologies éducatives de l'Université de Genève, formation postgrade consacrée aux technologies éducatives. Dans le cadre de cette formation, l'un de 


\section{Un classement fonctionnel}

Une comparaison des points de vue fonctionnel et sémiotique pourrait être intéressante : on pourrait en effet formuler l'hypothèse d'une correspondance entre des formes particulières de représentation et certaines fonctionnalités. Même si un classement fonctionnel des ILEIS pouvait s'avérer d'une grande utilité pour la suite de notre démarche, les deux points de vue - sémiotique et ergonomique - ne sont pas équivalents et de doivent pas nécessairement aboutir aux mêmes classifications. Les cas des icones et des boutons est de ce point de vue éclairant : la discrimination possède un poids important auprès des concepteurs d'interfaces; par contre du pointe de vue sémiotique, rien ne justifie que soient maintenues les deux classes.

Nous avons développé ces aspects dans un premier rapport de recherche et nous lui avons donné une suite récente dans un article à paraître (voir note 3 , à la page précédente). Aussi ne reviendrons-nous pas en détail sur ce point.

Nous avions à l'époque proposé trois classifications principales dont les deux premières conservent leur validité à la lueur des nos recherches actuelles. Il s'agit d'abord de l'importante distinction entre les icones de fonction et les icones de marquage. Les premières correspondent strictement à la définition donnée par Visual Basic, tandis que les secondes font partie des outils disponibles pour la mise en texte et l'ergonomie textuelle ${ }^{1}$. La deuxième différenciation porte sur les icones spécifiques et les icones génériques. Les premières sont uniques et représentent des fonctions qui n'appartiennent qu'à un seul environnement de travail, à un seul logiciel. Les secondes, quant à

nos enseignements (STAF13), porte sur les dispositifs éducatifs technosémiopragmatiques, sur la communication visuelle et sur les procédures de traitement et de visualisation de l'information. Le travail initial a été développé dans le cadre du projet de recherche "Projet Poschiavo" du Programme prioritaire "Demain la Suisse" financé par le FNRS suisse (requête 5004-47955, 1997/2000). Plusieurs critères proposés ont été validés expérimentalement dans le cadre d'un mémoire de troisième cycle (M. LINTZ, L'analyse des rapports texte/images dans les documents pédagogiques en biologie humaine: de l'imprimé au document électronique, Mémoire de 3ème Cycle, STAF, TECFA, Genève, 1998). Enfin, une classification des icones, de nature ergonomique cette fois, paraîtra prochainement : F. LOMBARD et D. PERAYA, "Éléments d'organisation gestuelle des icônes de logiciels et d'environnements informatiques standardisés", Recherches en communication, $\mathrm{n}^{\circ} 11,1999$.

1 Voir notamment S. CARo (op.cit.) ou G. NETCHINE-GRYNBERG, S. NETCHINE, Formation de structures sémiotiques graphiques par le jeune enfant ; mise en page, mise en texte, I et II, réunion du Groupe Théta, Cluny 9-11 sept. 1991, non publié. 
elles, représentent des fonctions générales, caractéristiques de tout logiciel ou de certaines familles d'"objets" ou d'opérations informatiques : aussi sont-elles normalement présentes dans de nombreux logiciels. Elles constituent un "vocabulaire visuel"1, dont la récurrence crée progressivement une culture informatique commune, un contexte d'arrière-fond partagé 2 . Tel est le cas par exemple des icones de sauvegarde et d'impression des fichiers qui sont représentées dans tous les systèmes et quasiment ${ }^{3}$ dans tous les logiciels de façon unique. Il s'agit de fonctions fondamentales de tout système informatique et ce fait suffit sans doute à expliquer leur généralisation.

La troisième classification concerne les icones-commandes et les icones-outils - les deux sous-classes des icones de fonction. Une icone-commande enclenche un processus dont l'exécution revient principalement au programme : les procédures de sauvegarde ou d'impression d'un fichier, déclenchées par l'icone correspondante, sont prise en charge automatiquement par le logiciel même si l'usager doit ou peut définir certaines variables - le format de sauvegarde, le nombre de copies ou l'ordre des pages, etc. Les icones-outils mettent à disposition de l'utilisateur un outil qui exige l'action sensori-motrice de l'utilisateur et l'intervention de son geste. C'est évidemment à ce niveau que les impératifs de l'ergonomie se font sentir le plus nettement et que notre classification s'est avérée la plus fragile.

1 Cf. Visual basic, op. cit.

2 J.R. SeArle, Les actes de langage. Essai de philosophie du langage, Paris, L'Harmattan, 1981 pour la traduction française. On pourrait aussi parler d'encyclopédie d'interprétation (J.-M. KLINKEnBERG, Précis de sémiotique générale, De Boeck, Bruxelles, 1996).

3 Il existe des exceptions, comme l'icone de sauvegarde dans l'éditeur HTML Hot Dog représentée par une bouée de sauvetage classique (blanche à bandes rouges) et régulièrement interprétée par les utilisateurs francophones comme l'icone donnant accès à l'aide en ligne... Elle semble n'avoir pas survécu. 


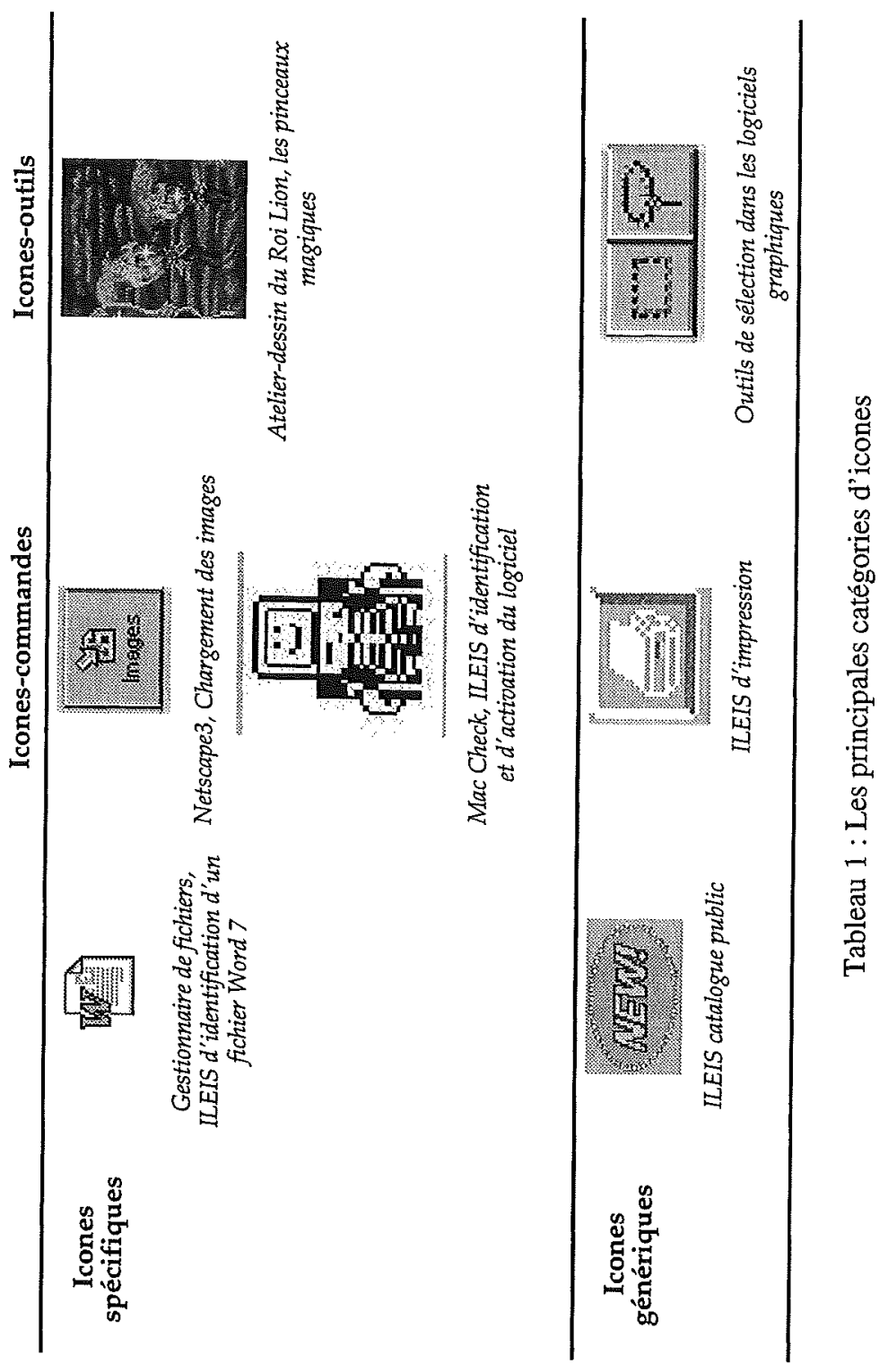




\section{La fonction désignative des ILEIS}

L'une des premières question est de savoir quel rapport unit le représenté et la représentation. Rappelons que l'icone n'existe jamais seule : elle est toujours accompagnée d'une description linguistique qui décrit la fonction, la commande, etc. que représente l'icone. La description se trouve disponible dans la bulle d'aide qui apparaît sous l'icone, dans le manuel, dans l'aide en ligne, ou encore dans les menus de personnalisation des barres d'outils. On ne peut donc analyser le rapport entre la fonction, la commande, etc. et leur ILEIS sans considérer l'énoncé linguistique qui leur correspond. En conséquence, et même si nous devrons revenir par la suite sur l'analyse du rôle de la structure et l'organisation linguistique de ces énoncés, une première approche nous permet de considérer l'icone et sa description comme deux unités d'information complémentaires se rapportant à une référence unique.

En transposant l'analyse des fonctions des unités d'information ${ }^{1}$ au cas des ILEIS, seules deux fonctions référentielles d'identification paraissent pertinentes. Rappelons brièvement leur définition et analysons leur bien-fondé dans l'analyse des ILEIS.

1. La fonction d'identification globale : l'icone assure une représentation globale du concept, de l'outil, de l'action représentés. La fonction est essentiellement désignative et l'icone se constitue en une unité autonome; en un iconogramme. La relation entre l'icone est sa référence est de l'ordre de l'identité. Les icones de l'imprimante ou de la disquette sont de ce type.

2. La fonction d'identification analytique: l'icone permet d'identifier le représentant en désignant certains traits caractéristiques de celui-ci ou les différentes étapes du processus à représenter. Chacun de ces motifs isolés peut être considéré comme un iconème. L'icone décompose, analyse et son fonctionnement rappelle le mode définition en compréhension. Telle est l'icone de l'utilitaire backup (Windows 3.1) qui décrit visuellement le transfert du contenu de l'ordinateur vers un coffre-fort, symbole par excellence de sécurité.

Tableau 2 : Les fonctions désignatives des ILEIS

1 D. PeRAYA, D. OTT, Projet Poschiavo : http:/tecfa.unige.ch/tecfa/research/poschiavo/ 
En ce qui concerne la fonction d'identification analytique, il faut signaler un cas particulier. Reprenons la série d'icones de mise en page (justification, centrage, alignement à gauche ou à droite). Chacune d'elle constitue bien évidemment une forme d'identification globale tant qu'on la considère pour elle-même, en tant qu'icone isolée. Par contre dès qu'on la restitue dans le contexte de sa série, c'est la fonction d'identification analytique qui semble le mieux convenir. En effet, chacune de ces quatre icones constitue l'un des aspects particuliers, un des états possibles de la fonction plus générale, du concept "mise en page".

\section{Nature sémiotique des ILEIS et de leurs composantes}

La dénomination commune de ces représentations paraît significative et le nom qui leur a été donné indique une conscience intuitive de leur nature sémiotique d'icone : ce sont des signes figurés, imitatifs. Pourtant les analyses même rapides que nous avons présentées cidessus ont montré qu'elles sont souvent plus complexes. Elles se composent en effet de plusieurs éléments iconiques, mais elles intègrent aussi des signes arbitraires: des éléments scriptovisuels, des signes linguistiques écrits - des graphèmes -, des signes typographiques, etc. Les ILEIS apparaissent donc parfois de nature composite, hétérosémiotique, comme le sont d'ailleurs de nombreuses représentations matérielles : publicités, messages télévisuels, panneaux de signalisation du code de la route, etc.

On le voit, dans tout signe analogique, il existe une part de convention de même qu'il peut exister une part d'analogique dans les signes symboliques. Ce ne serait donc pas l'opposition stricte entre motivation et arbitraire, entre analogique et digital, qui permet de distinguer ces deux classes fondamentales de signes : ce serait plutôt la prédominance de l'un de ces facteurs sur l'autre'. Les deux relations - iconique et symbolique - peuvent se manifester à des degrés divers dans un même signe : un graphique en secteurs, de type "camembert", est certes iconique puisqu'il présente de façon analogique une relation de proportionnalité entre différentes grandeurs, mais il comporte aussi une part de convention importante. On ne pourra d'ailleurs faire abstraction de la valeur indicielle de certains signes analogiques. Par

1 Sur ce point voir J.-P. Meunier et D. PERAYA, op. cit., Section 1, pp. 30-42 et Section 2, pp. 113-130 et 143-150. 
contre, une onomatopée est signe du langage verbal, un "symbole" dans la terminologie peircéenne. Pourtant, bien qu'elle doive être considérée comme arbitraire, elle comporte une part de motivation puisque l'onomatopée est par définition imitative. Mais en tant que signe linguistique, leur forme dépend entièrement des particularités phonologiques de chaque langue : le pigeon ne roucoule pas de la même façon en français, en anglais ou en italien...

Pour tenter de rendre compte de la diversité des représentations, les chercheurs ${ }^{1}$ ont longuement élaboré des échelles d'iconicité. Il revient à Moles d'avoir défini cette notion ainsi que la valeur iconale, à savoir la grandeur inverse de l'abstraction, autrement dit comme "la quantité de réalisme, d'imagerie immédiate contenue dans la représentation"2. Le plus souvent, ces échelles tentent de rendre compte de l'ensemble des représentations et des registres sémiotiques, des signes arbitraires - à iconicité nulle - à l'objet lui-même - à iconicité maximale. L'échelle à 12 degrés de Moles, sans doute inspirée dans sa démarche par le cône de l'expérience de Dale, qui date on s'en souviendra des années ' 60 , est de ce type. Les échelles n'ont pas que des avantages, tout au contraire. Il est très difficile en effet d'établir les limites entre les sous-catégories qui paraissent fortement arbitraires, ce que montrent les épreuves de validation inter-juges ${ }^{3}$.

Une perspective nouvelle a été récemment apportée par B. Darras qui aborde ces distinction du point de vue de la généalogie des familles de figures relevant de l'imagerie initiale et donc de leur morphogénèse : de l'icone du geste aux icones d'objets puis, finalement à celles de monde. Pour l'auteur, les processus de production d'images relèvent de deux types distincts de pensée :

a) d'une part, la pensée visuelle dont l'expérience optique et le visuel constituent le mode phénoménologique d'accès à l'information mais aussi son mode de traitement et de fonctionnement cognitif ;

b) la pensée figurative basée sur un matériel perceptif mais entièrement reconstruit par une économie cognitive. Autrement dit : "(..) la pensée visuelle construit son réseau de références, de vérification et de preuves dans le domaine le plus optique de l'expérience

1 Voir A. A. MOLES, op. cit. ; RICHAUdEAU, Conception et production de manuels scolaires, Paris, Retz/UNESCO, 1979/1981. D. PERAYA, M.-C. NYSSEN, op. cit.

2 A. A. MOLES, op. cit., p. 102.

3 D. PerayA, D. OTT, Projet Poschiavo. 
visuelle ; alors que la pensée figurative les construit dans le réseau et le jeu des catégories"'.

Il distinguera donc deux grandes familles de représentations, deux registres communicationnels - et sémiocognitifs, ajouterionsnous - distincts : les similis et les schémas qui comprennent notamment les iconotypes, les pictogrammes, les diagrammes, etc. Dans le second cas, ce sont les catégories, les résumés cognitifs et leur degré d'abstraction - de généralisation - par rapport au niveau de base, la typicalité, le relief cognitif, la figurativité, etc. qui jouent un rôle essentiel pour distinguer les sous-classes de représentations.

Compte tenu de notre corpus et de la simplification à laquelle donne la taille des icones, nous avons opté pour une classification à quatre degré s: le symbolique, le symbolique analogique, l'analogique conventionnel et l'analogique. Nous soutenons que ces quatre catégories simples suffisent à décrire exhaustivement la nature sémiotique de tous les éléments composant les ILEIS.

Aux deux extrémités de cette échelle, nous retrouvons des signes prototypiques : d'une part, le symbole peircéen, strictement conventionnel et arbitraire - le signe linguistique ou le langage formulaire et d'autre part, les représentations analogiques offrant avec l'objet représenté un fort degré d'imitation - dessin réaliste, et exceptionnellement la caricature et le dessin humoristique. Les deux catégories intermédiaires correspondent à cette zone "analogique-symbolique" que nous évoquions ci-dessus pour laquelle seule la dominante d'un des deux types sémiotiques peut servir de critère puisque l'on observe la présence d'éléments analogiques et imitatifs dans un signe arbitraire - symbolique analogique - et inversement la présence d'aspects conventionnels dans un signe à dominante analogique.

1 B. Darras, op. cit., p. 4. Cette opposition n'est pas sans rappeler celle proposée par Duval entre les représentations de type organique et celle de type sémiotique que nous avons évoquée ci-dessus. 


\section{ICONICITE MAXIMUM}

1. ANALOGIQUE : ressemblance, homomorphisme, (traitement figuratif sur base d'un donné perceptif)

2. ANALOGIQUE CONVENTIONNEL : homomorphisme, convention (traitement figuratif, signification conventionnelle)

3. SYMBOLIQUE ANALOGIQUE :

SYMBOLIQUE (arbitraire) mais incorporant valeur onomatopéique linguistique et/ou scripto-visuelle

4. SYMBOLIQUE : langue naturelle (signe linguistique) ou artificielle (langage formulaire)

ARBITRAIRE MAXIMUM

Tableau 3 : Échelle d'iconicité des ILIES et de leurs composants

Commentons brièvement les exemples que nous proposons pour chacune des catégories. L'icone de la paire de ciseaux - le "couper" de tous les logiciels standards - est une représentation strictement analogique : le représentant ressemble au représenté, il s'agit bien d'une contiguité par similarité selon la stricte définition de Peirce. Il s'agit d'ailleurs d'une icone générique, simple (un seul élément), monosémiotisée (ne mettant en œuvre qu'un seul registre sémiotique). Les exemples de ce type sont nombreux (l'imprimante, la disquette, etc...) et ne présentent aucune difficulté d'analyse ou de classification.

L'icone-commande qui permet d'accéder à la fonction prévisualisation du fichier avant son impression (print preview) issue de Word 7 est certes analogique dans la mesure où les deux éléments - deux sous-entités - qui la composent ressemblent à ce qu'ils représentent. Pourtant elle est aussi conventionnelle et sa signification globale est validé par convention, par l'ensemble des discours complémentaires qui l'accompagnent (la description verbale de bulle d'aide, le manuel en ligne ou imprimé, etc.). D'une part, la représentation de la feuille, le coin droit plié, est utilisée pour signifier le document ; on la côtoie 
d'ailleurs aujourd'hui dans d'autres contextes ${ }^{1}$, avec ou sans les lignes noires figurant les "portées d'écriture". Le fait que cette représentation se soit figée et soit réutilisée nous parait être un indice révélateur de son degré de conventionnalisation : elle deviendrait une entité sémantique stable, un icononème. D'autre part, la superposition de la loupe à la page - au fíchier - ne permet pas d'inférer "naturellement" la signification de l'icone puisque la loupe est utilisée habituellement - notamment dans les logiciels graphiques - comme icone-outil permettant d'agrandir certaines zones de l'écran. On comparera d'ailleurs avec l'ILEIS de la fonction zoom dans l'environnement de travail ouvert par la fonction "aperçu avant impression".

Un autre bon exemple de cette catégorie est l'icone du pinceau qui dans Word permet de "Reproduire la mise en forme". Il s'agit certes d'une représentation analogique d'un pinceau et tout le monde s'accorde sur ce point. Par contre, la signification qui lui est attribuée l'est par pure convention: aucune métaphore, aucun rapport de ressemblance entre le représentant et le représenté ne vient activer l'inférence nécessaire à sa compréhension réelle. Si l'on voulait absolument tenter un parallèle avec la théorie des actes de langage, on pourrait dire que cette icone constitue un acte de langage indirect conventionnel. Le processus de conventionnalisation peut donc affecter deux niveaux différents : celui d'une sous-entité de l'ILEIS - l'icononème - ou celui de l'icone elle-même - l'iconogramme.

La troisième icone est celle de l'aide contextuelle que l'on trouve dans les logiciels Microsoft de l'environnement Windows et Mac. Elle transforme le pointeur de la souris en un outil de désignation identique à l'icone. Elle comporte une sous-entité arbitraire, le point d'interrogation, qui appartient au langage écrit. Quant à la flèche, sa complexité nous intéresse et en fait la difficulté d'analyse ${ }^{2}$ : elle est à la fois conventionnelle et graphique, mais elle conserve une valeur indicielle : elle est la trace d'un geste, d'un mouvement ${ }^{3}$. Enfin dans

1 Si elle s'est imposée comme l'ILEIS de tout nouveau document sous Windows, on la retrouve, il est vrai avec ", dans l'icone d'identification des documents - des fichiers - Word, sur les fax et les photocopieuses pour indiquer le sens d'insertion des feuilles dans l'avaloir de la machine, etc.

2 M. LINTZ, Validation d'une grille d'analyse des rapports textes/paratextes dans les manuels de biologie et transposition à la publication électronique, Mémoire de Troisième cycle, STAF, TECFA, Genève, 1998.

3 En se référant aux classification des écritures figuratives, Darras parle dans ce cas de dactylogramme (op. cit., p. 153). 
le cas particulier de cette icone-outil, la flèche possède une valeur déictique, de désignation contextualisée. Elle désigne en effet, la partie de l'écran (le texte affiché, un élément de l'interface, etc.) qui fait l'objet de la question, c'est-à-dire le lieu que désigne l'utilisateur. Cette icone-outil s'avère indissociable de l'interaction : elle est l'équivalent parfait des embrayeurs linguistiques définis par Jakobson.

La dernière icone enfin, doit être considérée comme entièrement conventionnelle et arbitraire dans la mesure où elle présente une séquence de langage verbal écrit sans jouer aucunement sur l'aspect scriptovisuel qui aurait pu apporter une touche analogique supplémentaire.

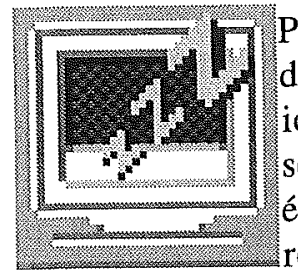

Prenons un dernier exemple plus complexe : l'icone d'un programme de veille d'écran pour Mac. Cette icone représente sur un écran d'ordinateur la séquence scripto-visuelle "zzz ": il s'agit bien évidemment d'un signe arbitraire composé de la répétition du graphème français /z/ (niveau 3, symbolique analogique). Au niveau de la langue orale déjà, il s'agit d'une onomatopée imitant le sifflement du dormeur. De plus, en tant que représentation scriptovisuelle, l'augmentation régulière de la taille des caractères mime l'augmentation régulière du volume sonore du ronflement du dormeur ou peut être, plus fondamentalement encore, l'augmentation volumétrique de sa cage thoracique. L'écran, quant à lui, est une représentation analogique de degré 1. Pourtant la superposition de la chaîne scriptovisuelle lui confère un référent anthropomorphe et métaphorique, celui du dormeur. Nous reviendrons sur ce mécanisme par la suite.

Résumons les différents caractéristiques - nature du signe et degré d'iconicité - de cette icone :

Langage oral

Langage scripto-visuel

\begin{tabular}{lll}
\hline $\begin{array}{l}\text { Aspects conven- } \\
\text { tionnels }\end{array}$ & $\begin{array}{l}\text { Suite de phonèmes de la } \\
\text { langue }\end{array}$ & Suite de graphèmes \\
\hline Aspects imitatifs & $\begin{array}{l}\text { Sifflement/ronflement } \\
\text { en crescendo, mouve- } \\
\text { ment respiratoire }\end{array}$ & $\begin{array}{l}\text { Augmentation régulière de } \\
\text { la taille des graphèmes }\end{array}$ \\
\hline
\end{tabular}

Tableau 4 : Analyse des composantes signifiantes un signe hétérosémiotique 
Enfin, disons que ce signe scriptovisuel de type symbolique analogique " $\mathbf{z Z Z Z Z Z " ~ s ' e s t ~ q u a s i m e n t ~ f i g e ́ ~ d a n s ~ l e ~ l a n g a g e ~ d e ~ l a ~}$ bande dessinée où il constitue aujourd'hui un élément sémantique dont la forme et le contenu paraissent stabilisés. Il constituerait un autre cas intéressant d'iconème.

On le voit, la nature hétérosémiotique des icones participe à leur complexité. Pourtant ce n'est pas le seul élément : leur structure y contribue aussi largement.

\section{Principes d'organisation sémiopragmatique des ILEIS}

\section{Le degré de complexité structurelle des ILEIS}

Techniquement, nous proposerions la distinction entre l'inconème et l'iconogramme, ou plus simplement l'ILEIS. Le premier consiste en un sous-élément constitutif de l'ILEIS, particulier et isolable : la loupe de l'icone "aperçu avant impression" dans Word par exemple (voir ci-dessous). Par contre, dans de nombreux logiciels de dessin la loupe constitue à elle seule une icone, celle qui commande la fonction d'agrandissement. Qu'existe la possibilité d'utiliser certaines représentations soit comme entité, soit comme sous-entité, indique clairement le processus de décontextualisation et de généralisation auquel sont soumises ces unités dans un processus de systématisation. On peut donc penser que nombreux iconèmes sont à l'origine des iconogrammes qui progressivement, dans l'usage, ont conquis une certaine autonomie leur permettant d'être réinvestis dans une composition complexe.

Les ILEIS, nous venons de le voir, sont souvent composites : beaucoup sont constituées de plusieurs éléments dont la nature sémiotique est parfois hétérogène. Enfin, certaines icones sont isolées tandis que d'autres s'intègrent dans une série comme les quatre icones de mise en page (aligner à gauche, à droite, justifier, centrer), les deux icones qui permettent d' "annuler" les dernières opérations ou de les "restituer" comme c'est le cas dans de nombreux logiciels tels que Word. L'icone prend alors son sens par rapport au co-texte immédiat, par opposition aux autres éléments de la série.

Le nombre d'éléments composant une icone et leur niveau (entité, sous-entité) va nécessairement avoir une influence sur la 
structure et l'organisation interne des ILEIS. Nous avons déjà indiqué que certains éléments composant une icone pouvaient s'autonomiser au point de pouvoir être intégrés dans la composition d'autres icones ou encore d'être utilisés seuls comme une icone à part entière. Tel était le cas de la loupe qui entre dans la composition de l'icone "aperçu avant impression" déjà citée et par exemple dans celle de l'icone de connexion à Internet dans l'environnement Windows (une loupe superposée à un globe terrestre). Enfin nous l'avons dit, la loupe peut être utilisée seule (fonction zoom). Ces exemples nous ont fait adopter respectivement les termes d'iconème, dans le cas d'une sous-entité, et d'iconogramme, dans le cas de l'entité.

L'ILEIS de l'imprimante, souvent citée comme exemple, pose de ce point de vue un problème. Dans un premier temps nous avions considéré qu'elle était composée de deux sous-entités : l'imprimante elle-même et, en train de sortir de la machine, la feuille de papier avec ses traits noirs figurant quelques lignes d'écriture. Notre position a changé : nous sommes enclin à considérer globalement cette ILEIS, comme l'icone de l'imprimante en train d'imprimer. Les traits horizontaux, les lignes d'écriture, ne sont interprétables comme telles que dans le cadre de cette ILEIS. Il en va de même pour la portion de feuille à moitié imprimée, à moitié sortie de la machine. On pourrait dire la même chose de la disquette de l'ILEIS de sauvegarde : les détails figurés qui permettent de la reconnaître comme la représentation d'une disquette font partie intégrante de la représentation globale de la disquette et ne peuvent en être séparés.

On devrait donc considérer quatre niveaux de complexité au sein des ILEIS. Au premier niveau, les éléments figurés non significatifs en dehors de l'ILEIS : il constituent les traits saillants, les attributs prototypiques et figurables de l'objet ou de l'action représentés. Ils appartiennent à un tout et n'ont de signification en dehors de lui. Ils participent donc à l'identification de l'ILEIS. Au deuxième niveau, nous rencontrerions des iconèmes, des motifs - ou encore des figures - identifiables et autonomes, autrement dit dotés d'une signification propre (fonction d'identification globale). Le troisième niveau serait constitué par celui de l'ILEIS. Enfin, les icones qui entrent dans une série plus ou moins étendue et qui se comprennent en référence au cotexte définissent le quatrième et dernier niveau de complexité.

Nous retiendrons donc pour rendre compte cette diversité les critères descriptifs suivants : 
1) le fait que l'ILEIS soit simple (une entité composée de traits saillants) ou composite (plusieurs sous-entités) ;

2) le nombre d'iconèmes ou de sous-unités composant l'ILEIS, cinq étant le maximum observé ;

3) le caractère unique ou sériel de l'ILEIS.

Ces trois catégories détermineraient des niveaux d'organisation que nous qualifierons respectivement d'intra-iconique (au sein de l'ILEIS considérée comme entité), d'inter-inconèmique (entre les iconème de l'ILEIS), inter-iconique dans le cas des icones sérielles.

\section{La syntaxe topologique des ILEIS}

Dans le cas d'icones complexes et composites, nous avons débattu longuement de la façon d'en distinguer et d'en isoler les différents composant. Quels sont dès lors leurs mécanismes de structuration et d'organisation sémiotiques internes ? Comment sont organisées entre elles les sous-entités, les figures ou encore les iconèmes ? Ces formes d'organisation s'avèrent-elles suffisamment différentes pour distinguer les ILEIS des iconotypes? L'analyse de l'organisation visuospatiale et de la syntaxe iconique devrait pouvoir offrir une base descriptive permettant de donner une première réponse à ces différentes questions.

Dans son étude sur l'imagerie initiale, Darras étudie l'organisation des iconotypes sous trois angles particuliers :

1) les catégories topologiques, c'est-à-dire, les zones privilégiées de l'espace de représentation notamment à partir des concepts de centrement, de gravité, de verticalité, d'horizontalité, de latéralisation, etc.

2) la topologie interne, i.e. l'influence de ces catégories, de ces substrats spatiaux, sur les espaces locaux que constituent les figures ;

3) la topologie externe, i.e. l'étude des interrelations entre les différentes figures.

Nous ne suivrons pas cette démarche même si nous la rencontrerons sur certains points. Par exemple parmi les modes d'organisation topologiques définis par l'auteur, le voisinage, l'étagement et peutêtre la juxtaposition correspondent à ce que, tout au début de cette étude, nous avions appelé par analogie avec les catégories linguis- 
tiques une forme d'organisation de type "apposition"1. Nous discuterons ci-dessous la différence entre ces différentes formes d'organisation topologiques.

Ajoutons encore que cette analyse ne concernerait en toute logique que les ILEIS composites. Cependant, puisque nous avons pour objectif de décrire les structures générales des ILEIS, il nous faudra proposer une première catégorie comprenant les icones simples, non composites et une autre constituée par les ILEIS de type sériel.

A partir du corpus recueilli et des résultats de l'étude exploratoire, six catégories suffisent à décrire les différentes modalités d'organisation et la syntaxe des ILEIS. Nous retiendrons les ILEIS simples, caractérisées par l'absence d'une organisation entre différentes entités et les ILEIS composites, structurées selon les modalités suivantes : superposition, apposition, articulation, inclusion et métaphorisation.

\section{Les icones simples}

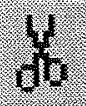

Nous prendrons comme premier niveau d'organisation, les icones simples, non composées. Il s'agit du degré zéro de la syntaxe iconique puisque il n'existe à proprement parlé aucune organisation, l'ILEIS - l'iconogramme - se composant d'un unique motif, d'un seul iconème. Cette icone est conçue sans doute sur la base d'un donné perceptif, mais surtout selon un résumé cognitif mettant en évidence les principaux attributs saillants ou représenté. L'ILEIS de la fonction "couper" ou celle d'impression appartiennent à cette catégorie.

1 Nous avons hésité longuement à utiliser le terme de parataxe. Celle-ci s'oppose à la subordination et à la coordination dans la mesure où les phrases sont "juxtaposées sans expliciter par une particule de subordination ou de coordination le rapport de dépendance qui existe entre elles dans un énoncé, dans un discours, dans une argumentation" (J. Dubors et al., Dictionnaire de linguistique, Paris, Larousse, 1974 , p. 356.). Apposition nous a paru surtout plus facile à utiliser dans ce texte : l'adjectif apposé nous paraît en effet plus léger que "parataxique". 


\section{Les cas de superposition}

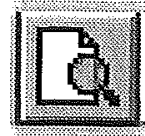

L'iconème de la loupe se superpose à celui de la page et la superposition conserve à la loupe sa transparence. Nous l'avons dit, c'est par convention que l'association de ces deux sous-entités confère à l'ILEIS sa signification "aperçu avant impression". Pourtant, et cela peut paraitre paradoxal, la représentation globale correspond assez bien à ce que nous savons d'un droitier qui lirait un texte avec une loupe : nous y reconnaissons à travers la position de la loupe sur la page, le geste et la main du lecteur. La représentation globale, l'association "loupe sur feuille" apparaît fondamentalement mimétique, voire naturelle, présentant la trace reconnaissable de postures et d'un gestuel.

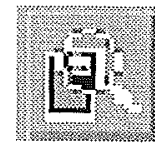

Cette seconde ILEIS, la loupe de l'environnement de travail "aperçu avant impression" dans Word, présente un autre cas de superposition par transparence et pourtant elle est assez différente de la première. D'abord, le rapport de taille est inversé : dans ce cas c'est la loupe qui est plus grande. Ensuite, la transparence laisse entrevoir le texte grossi par l'effet loupe alors que dans le premier cas, la transparence semblait caractériser par ses reflets la lentille et l'optique, donc le potentiel de grossissement que possède l'outil. Dans le premier cas la visée porte sur l'action de grossir tandis que dans le second, l'ILEIS exprime directement l'effet de grossissement.

Cette différence est intéressante car elle montre que, même si dans les deux cas, la superposition peut se baser sur un mécanisme analogique, reprenant dans sa structure la position quasiment naturelle qu' aurait un lecteur droitier utilisant une loupe, de nombreux éléments conventionnels viennent se surimposer afin de spécifier des significations différentes.

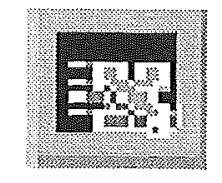

Dans le cas de cette ILEIS qui insère une feuille de calcul Excel, la superposition semble répondre à une logique différente. Tout d'abord, la superposition implique l'opacité de l'élément surajouté, ce qui correspond d'ailleurs au mode de composition par superposition dominant. Ensuite, l'association paraît bien plus conventionnelle que naturelle bien que le coin inférieur droit d'un document, d'un tableau 
soit souvent celui de la signature, donc de l'identification du propriétaire.

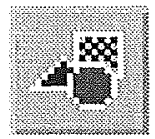

L'ILEIS d'insertion d'un dessin dans Word par exemple est composée d'un cercle, d'un triangle et d'un carré en superposition : le procédé de superposition paraît ici tout à fait conventionnel et leur ordre semble déterminé essentiellement par des raisons de composition : si l'on regarde cette icone, on se rendra compte du rôle joué par les contraintes du centrement, les diagonales et les médianes. Les trois iconèmes semblent avoir été choisis en tant que traits saillants du concept "dessin" ce que pourrait confirmer le fait que le trait, le rectangle et l'ellipse dont dérivent respectivement le triangle, le carré et le cercle, sont les trois outils graphiques de base de ce type de logiciel.

\section{Les cas d'apposition}

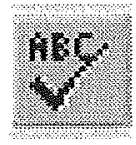

L'icone du correcteur orthographique que l'on trouve dans de nombreux logiciels constitue un des cas de structuration sur le mode de l'apposition: les iconèmes, quelle que soit leur nature sémiotique (symbolique ou analogique), sont simplement juxtaposés sans qu'aucune marque explicite ne permette de déterminer le lien de détermination, le lien logique qui les unissent. Et pourtant de toute évidence, ce rapport de subordination entre ces sous-entités existe bel et bien. C'est donc bien à la terminologie linguistique que nous nous référons. Du point de vue topologique, dans ce cas-ci les sous-entités sont contiguës, mais c'est loin d'être la règle : elles peuvent être, selon l'expression de Darras, "voisines", laissant entre elles un espace libre.

Nous conserverons à cette rubrique le terme générique d'apposition pour désigner les différentes relations qu'entretiennent les sousentités : contiguité, voisinage ou encore étagement.

\section{Les cas d'articulation}

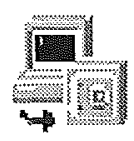

Ce n'est évidemment pas la superposition qui est caractéristique de cette ILEIS (le programme de back up sous Windows), mais bien l'introduction d'un 
organisateur logique et/ou relationnel conventionnel - dans ce cas-ci, la flèche - entre les deux iconèmes. Le statut de la flèche, nous l'avons dit, est complexe : elle est conventionnelle, mais elle est aussi mimétique et indicielle.

L'articulation constitue un mode de construction spécifique et très différent de la simple apposition ou puisque la relation est cette fois explicite et clairement définie. On sait que l'image, au sens large, se distingue du langage verbal notamment par son incapacité à signifier certaines modalités telles que la négation ou l'articulation logique ${ }^{1}$. Une image postule l'existence de l'objet représenté, elle est affirmation de ce qu'il a existé ou de qu'il existe encore. Aussi faut-il pour signifier la non existence surajouter un signe conventionnel : la croix qui barre l'objet et signifie formelle son interdiction (la cigarette barrée pour l'interdiction de fumer, par exemple) ou alors l'utilisation du langage verbal dont le fameux "Ceci n'est pas une pipe" de Magritte constitue aujourd'hui le canon.

Un articulateur conventionnel peut être introduit entre des iconèmes superposés ou apposés. Les cas d'articulation constituent donc une façon d'expliciter le lien de détermination entre les différentes sous-entités de l'ILEIS, quel que soit leur mode d'association.

\section{Les cas d'inclusion}

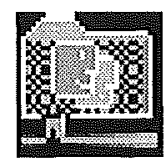

Cette ILEIS appartient à l'univers Mac (Système d'opération du Mac-OS, domaine de partage de fichiers, mise en réseau Apple-Share) présente un cas d'inclusion par superposition sans transparence. $\mathrm{Ce}$ procédé conduit à l'intégration des différents iconèmes en une seule unité globale, en un iconogramme selon le principe de condensation. Les cas d'inclusion - l'inclusion constitue un des modes de composition par métaphorisation - sont extrêmement rares et c'est d'ailleurs le seul cas que nous ayons pu répertorier. On peut donc se demander quelle est la pertinence de cette catégorie et s'il vaut la peine de la maintenir.

Provisoirement, nous répondrons que oui. Dans cet ILEIS, dont on perçoit cependant la visée métaphorique, l'inclusion ne réalise pas totalement l'intégration puisqu'elle se combine avec le procédé de

1 D. PerayA, "Des mots et des images, Chronique d'images", Journal de l'enseignement primaire, $\mathrm{n}^{\circ} 49$, mars/avril 1994, pp. 22-25. 
superposition, ce que renforce encore la non transparence des iconèmes "rapportés". On observe donc un phénomène de conservation des iconèmes et le fichier de fond reste déterminant. La différence entre inclusion et métaphorisation, bien que sa limite soit difficile à tracer, se situerait donc dans le degré d'intégration entre les iconèmes : lorsque "le tout vaut plus que la somme de ses parties", la métaphorisation serait complète.

\section{Les cas de métaphorisation}

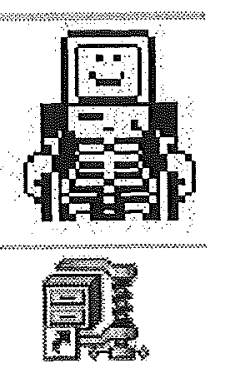

L'ILEIS du logiciel Mac Check réalise complètement l'intégration des différents iconèmes à travers la métaphore anthropomorphe et médicale de l'ordinateur radiographié. Dans les cas de métaphorisation, la logique de structuration introduit un homomorphisme entre la structure de l'ILEIS et celle de la métaphore elle-même. Le logiciel qui analyse le disque dur doit être compris dans les termes du médecin radiologue qui procède à une radio du thorax de son patient. Le logiciel de compression de fichiers Winzip doit être interprété comme un étau qui compresse un meuble de rangement à tiroirs. Autrement dit, la métaphore constitue un aspect rhétorique mais fondamentalement un aspect cognitif : elle est un principe structurel de compréhension : "un concept est métaphoriquement structuré en termes d'autres concepts"l.

\section{Organisation syntaxique analogique ou conventionnelle}

En même temps que nous décrivons les modalités d'organisation syntaxique des ILEIS, nous devons observer que celles-ci pouvaient relever de deux logiques différentes. Dans le cas de l'ILEIS "aperçu avant impression", nous avons parlé d'un procédé de superposition de nature mimétique ou analogique puisqu'il renvoie à des aspects

1 G. LAKoff et M. Johnson, op. cit., p. 24. 
analogiques, mimétiques, posturaux du représenté. Pourtant nous avons dû nuancer cette affirmation : la comparaison avec l'ILEIS de la loupe laissait en effet apparaitre des éléments conventionnels au sein d'une organisation principalement analogique.

Par contre, dans les autres cas - sans doute les plus nombreux -, les iconèmes se distribuent selon les critères topologiques qui sont analysés et discutés ci-dessous : dessus/dessous, avant-plan/arrièreplan, haut/bas, gauche/droite. Dans ces cas là, le mode d'organisation syntaxique doit être considéré comme plus conventionnel qu'analogique même si une discussion détaillée de ces catégories nous obligera à nuancer cette position. L'essentiel n'est pas d'avoir des catégories tranchées, mais de montrer que deux logiques sont à l'œuvre et qu'elles peuvent même coexister au sein d'une même ILEIS.

Bien évidemment, ces deux caractéristiques de l'organisation syntaxique s'appliquent aux différents principes d'organisation que nous avons inventoriés ci-dessus. Dans le tableau ci-dessous, nous présentons sans aucune prétention à l'exhaustivité quelques exemples non ambigus.

\begin{tabular}{ccc}
\hline Principe d'organisa- & $\begin{array}{c}\text { Nature du principe } \\
\text { tion syntaxique }\end{array}$ & d'organisation syntaxique \\
\hline & Superposition & Principalement analogique \\
\hline & Apposition & \\
& & \\
\hline
\end{tabular}

Tableau 5 : Quelques exemples d'analyse croisée selon les paramètres d'organisation syntaxique 


\section{Les caractéristiques topologiques ${ }^{1}$}

\section{Le centrement : carré graphique, carré visuel}

Rappelons tout d'abord que toutes les représentations des ILEIS ne sont inscrites pas dans un espace cadré. Le cadre est sans aucun doute la première catégorie topologique privilégiée de tout espace de figuration. On connaît l'importance graphique et sémiotique de cet espace délimité, de ce "plan originel appelé à porter l'œuvre" selon l'expression de W. Kandinsky. Quel que soit leur champ disciplinaire de référence, tous les travaux confirment la prégnance de cet espace cadré de départ. A l'appui de cette thèse, Darras rappelle les travaux des critiques et théoriciens d'art - R. Passeron, R. Arnheim -, ceux de sémioticiens - M. Shapiro - ou encore les recherches concernant les singes peintres - H. Schiller, B. Rensh, T. Lenain, D. Morris ${ }^{2}$. Par ailleurs, les travaux des psychologues S. Grynberg et G. NetchineGrynberg ${ }^{3}$ ont montré que la structure de la page, sa structure orthogonale et les portées d'écriture s'imposent progressivement au jeune enfant comme des structures sémiotiques fondamentales : la feuille blanche, dirons-nous, acquiert le statut de page ${ }^{4}$.

Les boutons graphiques sont par excellence des espaces cadrés dont la forme et les dimensions contraignent fortement la structure figurative de l'icone. Ils sont d'ailleurs carrés contrairement à la plupart des surfaces graphiques de départ qui sont rectangulaires et, en majorité orientées, horizontalement (plus de $80 \%)^{5}$. Ce format carré explique selon nous l'utilisation quasiment exclusive du centrement $^{6}$ comme principe de construction topologique de l'icone. L'icone de commande "couper" qui représente une paire de ciseaux constitue un bon exemple puisque l' axe de rotation de lames se situe à la croisée des diagonales du carré, matérialisées quant à elles par les lames et les poignées. Le nez du chien dans l'ILEIS du logiciel Fetch se trouve lui aussi au centre de gravité du carré. Toutes les icones qui

\footnotetext{
1 Nous avons volontairement omis dans ce texte de prendre en considération les modalités de représentation $2 \mathrm{D}$ et $3 \mathrm{D}$.

2 W. KANDINSKY cité par B. DARRAS, op. cit., p. 173. Tous les auteurs cités le sont à la même page.

3 G. NETCHINE-GRYNBERG, S. NETChINE, op. cit.

4 D. Peraya, "Une révolution sémiotique", Cahiers pédagogiques, n³62, mars 1998, pp. 26-28.

5 B. DARRAS, op. cit., p. 177.

6 Ibid., pp. 174-179.
} 
nous servent d'exemple dans ce texte offrent le même type de composition.

Enfin, les icones non cadrées - les dossiers, les raccourcis vers un programme ou les alias, les icones "système" comme la poubelle, le poste de travail, etc. - s'inscrivent naturellement dans un carré "visuel" selon l'expression de A. Mante ${ }^{1}$, déterminé par leur format de base. Les deux icones présentées dans le tableau ci-dessous, celle du programme de compression Winzip et celle du programme de back $u p$, font apparaître un centre, à la croisée des diagonales : il s'agit respectivement du coin inférieur droit du meuble de classement et du coin supérieur gauche du coffre-fort. Que l'icone s'inscrive dans un cadre dessiné ou simplement reconstitué visuellement, le principe topologique du centrement s'impose puisque dans les deux cas la surface de l'icone est carrée.

On pourrait d'ailleurs se demander s'il existe une différence entre les icones cadrées et celles qui ne le sont pas et, le cas échéant, quel en serait l'impact. Les icones non cadrées, plus que les autres, paraissent en effet mobiles : l'utilisateur peut les déplacer, les grouper, les structurer au gré de sa fantaisie et/ou des ses habitudes de travail. On peut faire l'hypothèse que chacun de ces deux types possède une sémantique particulière. En conséquence, chacun pourrait induire de la part de l'utilisateur une attente et un comportement différents, par exemple respectivement une visée de représentation ou une visée d'objet. Si c'était le cas, un bon indicateur pourrait en être le gradient de personnalisation relative - par rapport à la configuration standard - des barres d'outils et du bureau bien que l'on puisse supposer l'importance du rôle joué par le degré de familiarité avec l'outil informatique, les tâches et les stratégies de travail, etc. Sans que nous soyons capable actuellement de donner une réponse à cette question, nous la croyons pertinente : le Système 8 du Mac permet par exemple d'afficher les mêmes commandes sous l'un ou l'autre format, bouton ou icone et de surcroît comme une liste.

Nous venons de le voir, le centrement semble même constituer le mode de structuration par défaut des ILEIS. En toute logique, il ne faudrait donc n'en tenir compte que dans les éventuels cas constituant une exception à cette règle.

1 A. MANTE, La composition en photographie, Paris, Dessain et Toltra, 1971. 


\section{Les rapports de détermination topologique}

Les autres catégories topologiques sont constituées par les oppositions classiques: gauche/droite, haut/bas, dessus/dessous, devant/derrière. Il est une catégorie supplémentaire, non topologique, qui semble présenter le même type de fonctionnement : il s'agit du rapport de taille relative entre les deux éléments que nous avons déjà évoqué à propos de l'ILEIS de la loupe dans Word. Ces oppositions définissent les rapport de détermination réciproques entre les différents iconèmes d'une ILEIS composite. Ils joueraient donc un rôle important dans l'organisation topologique des sous-entités et contribuerait à leur organisation syntaxique au sein de l'ILEIS. Par opposition à la logique analogique dont nous avons dit qu'elle est l'un de deux facteurs à régir l'organisation syntaxique, on pourrait supposer que les catégories topologiques soient à la base de la seconde, la logique conventionnelle. Il ne semble cependant pas y avoir de véritable régularité dans la disposition topologique des iconèmes. Il est vrai que seule une étude statistique d'un corpus pourrait permettre de dégager les éventuelles régularités en ce domaine : le caractère exploratoire de notre analyse impose ici ses limites.

Or, ces catégories - de nombreuses recherches de psychologues, de plasticiens et de théoriciens de l'art l'attestent - semblent investies d'une sémantique relativement stable, fondée partiellement sur notre expérience de la gravité, de la verticalité, de l'horizontalité et de la latéralité. A l'appui de cette thèse Darras cite de nombreuses recherches sur le dessin d'enfant (Wallon et Lurçat), sur les sujets atteints de lésions cérébrales (Gardner et Vigouroux, par exemple) ${ }^{1}$. Par exemple, il rappelle la tendance spontanée des sujets occidentaux spécialistes et novices à préférer dans les compositions non figuratives celles "ayant les masses plus importantes vers le bas et par conséquent, plus 'légères' vers le haut"2. Nous avons récemment proposé une synthèse des conceptions actuelles sur cet important problème à partir de références différentes, mais entièrement convergentes $^{3}$. On sait aussi que la tendance générale est de

1 H. WALLON, L. LURÇAT, "L'espace graphique de l'enfant", Journal de psychologie, $n^{\circ}$ 4, 1959, pp. 427-453. Cités par B. DARRAS, op. cit., pp. 184 et 195.

2 E. Winner, J. DIon, E. RosenblatT, H. GARDNER, "Do Lateral or Vertical Reversals Affect Balance in Painting", in Visual Art Research, 1987. Cité par B. DARRAS, op. cit., p. 186.

3 D. Peraya, J.-P. MEunier, "L'image et le mot : vers une sémiotique cognitive", VOIR, n 16 , mai 1998 , pp. 16-27. 
positionner le personnage le plus grand à gauche et le plus petit à sa droite.

On sait que la représentation de l'objet ne se fonde pas exclusivement sur la perception visuelle et sur l'analogie puisqu'elle intègre des propriétés non visuelles : symboliques et langagières, nous venons de le dire, mais aussi indicielles, liées à notre expérience sensorimotrice, à notre expérience des lois physiques du monde comme l'avaient déjà noté, par exemple, Wallon et Piaget ${ }^{1}$.

Cette prise de position relativise fortement le caractère conventionnel que nous avions attribué par principe aux catégories topologiques. Or, la question de leur sémantique est extrêmement importante puisque, dans les cas d'apposition et de superposition conventionnelle, l'explicitation des rapports de détermination entre les iconèmes pourrait reposer principalement sur cette base.

Une autre voie serait d'analyser les descriptions linguistiques qui accompagnent les ILEIS.

\section{Message analogique et verbal}

A titre d'exemple, prenons la description, le label, de l'icone de Word qui permet d'insérer une feuille de calcul Excel. Son label la définit d'ailleurs strictement en ces termes : "Insérer feuille de calcul Excel". L'icone correspond à une action, à un concept, complexe dont la formulation linguistique correspond déjà à une phrase entière décomposable en :

- un groupe nominal (sujet en effacement) ;

- un groupe verbal composé d'un groupe nominal ("la feuille") et de ses expansions ("de calcul, Excel").

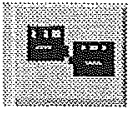

La complexité de la structure dépend bien sûr de celle des expansions. Que dire alors de cette autre icone de Word proposée dans la barre d'outils "insertion"qui se définit comme suit : "Insère une base de données externe dans le document actif" ! Le parallélisme avec le langage naturel est sans doute une voie méthodologique peu conseillée et toute l'histoire de la sémiotique a montré les limites d'une telle démarche quand elle était suivie strictement. Mais puisque l'ancrage linguistique est indispen-

1 Voir M. DENIS et M. DE VEGA, op. cit., 1993. 
sable à l'intelligibilité des icones, on pourrait se demander quelle serait éventuellement l'influence du degré de complexité de la définition verbale sur les mécanismes de construction sémiotique de l'icone. Qu'il existe un rapport pourrait constituer une hypothèse forte, encore que dans le cas précis des logiciels en langue française, l'analyse risque de pâtir d'un biais important puisqu'il s'agit d'une traduction et que le travail de conception des ILEIS et de leur composition a été réalisé selon toute vraisemblance par rapport à la langue anglaise.

Nous ne partagerons pas les positions de A. A. Moles lorsqu'il tente de trouver au langage des schémas des correspondances avec le langage naturel: "le schéma est le produit d'un acte de communication (...). Par là, il suggère un parallélisme avec le langage dont il possède toutes les caractéristiques : des signes, un vocabulaire, une syntaxe, une logique, une intelligibilité" 1 . Par contre, comme nous l'avons déjà suggéré, la comparaison des registres sémiotiques et l'analyse de la convergence sémantique - au sens de Duval semblent une voie intéressante pour tenter d'articuler les messages de nature iconique et verbale.

Il serait important, mais cette fois encore le caractère exploratoire de cette étude n'autorise aucune réponse, de voir s'il existe des correspondances entre les relations topologiques et des structures sémantiques de type prédicatif - sujet/prédicat ou thème/commentaire - ou d'autres modèles analogiques dont la sémantique cognitive pourrait, par exemple, constituer le modèle descriptif. On se souviendra que pour Langacker

les expressions linguistiques donnent lieu à des images variées d'une même situation objective. Les images diffèrent selon les aspects de la situation qu'elles sélectionnent, le degré de saillance de ces aspects, leur degré d'abstraction, la perspective sous laquelle ils sont vus, etc. Les conventions lexicales et grammaticales seront là précisément pour fournir les expressions nécessaires à sa structuration ${ }^{2}$.

A. A. MOLES, op.cit., p. 102.

2 R. LANGACKER, Foundations of Cognitve Grammar, Stanford, Stanford University Press, 1987. Cité par J.-P. MEunIER, "Connaître par l'image", Recherches en communication, $\mathrm{n}^{\circ} 10,1998$. 
Les deux icones "aperçu avant impression" et "loupe" dans cet environnement semblent supposer que cette hypothèse pourrait avoir un sens.

\section{Procédés rhétoriques}

Nous rappellerons ici les deux procédés rhétoriques que nous avons déjà abondamment évoqués à l'occasion de l'analyse des différentes icones qui jusqu'ici nous ont servi d'exemple : la métaphore et la métonymie.

\begin{tabular}{ll}
\hline Associativité des images & Discours rhétorique \\
\hline Principe de continuité & Métonymie \\
Principe de similarité & Métaphore \\
\hline
\end{tabular}

Tableau 6 : Les principes d'associativité des images dans le discours et le rêve

La signification de nombreuses icones se fonde sur un processus métonymique parfois complexe, puisque dans certains cas trois opérations successives sont nécessaires pour passer du sens littéral, de la valeur désignative (ceci est une disquette) à la signification réelle : commande de sauvegarde du fichier en cours, quel que soit le support de stockage. D'autres enfin, celle du chien fidèle (Fetch), du programme de veille, ou du programme d'analyse du disque ( $M a c$ Check), utilisent la métaphore comme principe de compréhension. Elles correspondent strictement à la définition que donnent Lakoff et Johnson des métaphores structurelles : "un concept est métaphoriquement structuré en termes d'un autre concept"1. Métonymie et métaphore peuvent, nous l'avons montré, coexister au sein d'une seule et même icone. Dans ce cas, la métonymie porte sur des éléments - un iconème - de la représentation globale de l'icone.

\section{Place de l'énonciateur}

Dans le cadre des dispositifs d'énonciation médiatique, il faut bien sûr identifier les marques de relations intersubjectives et d'interpellations qui caractérisent le registre du discours.

1 G. LAKOFF et M. JoHnSON, op. cit., p. 24. 
Les icones sont-elles aussi construites comme des dispositifs d'énonciation, incluant une dimension pragmatique, assignant au lecteur une place dans le dispositif qui relève soit du mode du récit soit de celui du discours. La plupart des icones appartiennent au mode du récit. Les rares occurrences d'ILEIS qui présentent les caractéristiques du discours sont celles qui reposent sur une métaphore anthropomorphe : le chien de Fetch, l'ordinateur patient radiographié, etc.

\section{Conclusions}

Notre analyse n'a, nous l'avons dit, qu'un caractère exploratoire. Elle n'offre encore aucune validation statistique et fait abstraction de plusieurs dimensions dont on ne peut, au vu du développement des environnements informatiques, ignorer l'importance : d'une part, le mouvement et l'animation et d'autre part, les formes de représentation de l'espace tridimensionnel. Enfin, nous n'avons pas approfondi les rapports d'organisations syntaxique et topologique entre les icones sérielles. Malgré ces limites, nous pensons avoir répondu à de nombreuses questions posées dans notre introduction.

Tout d'abord, nous pensons avoir fait la démonstration qu'il existe une "grammaire" ILEIS et qu'elle est relativement complexe et stable. Rappelons quelques observations majeures :

1) La nature sémiotique des ILEIS et/ou de leurs composantes oscille entre l'analogique et le symbolique. Pourtant, il est souvent difficile de trouver des ILEIS exclusivement représentatives de l'un ou l'autre de ces deux pôles : dans bien des cas l'analogique se mêle au symbolique et vice versa. Le scriptovisuel, c'est-à-dire le langage verbal écrit, comporte souvent une dimension visuelle qui ouvre la voie à la part de conventionnel. Inversement, l'analogique comporte quasiment toujours une part de convention, comme 1'a montré la comparaison entre les icones "aperçu avant impression" et "effet zoom" de l'aperçu avant impression.

2) Il existe des ILEIS simples et d'autres complexes. D'une part, ces ILEIS sont composites, c'est-à-dire qu'elles sont composées de plusieurs éléments, que nous avons appelés des iconèmes. D'autre part, ces derniers peuvent être de nature sémiotique différente et se situer à divers degrés d'une échelle d'iconicité allant du plus arbitraire, le langage verbal écrit, au plus analogique, les icones au 
sens peircéen. Enfin, certaines icones peuvent entrer dans une série plus large et ne prennent dès lors leur sens que par rapport au cotexte.

3) Cette analyse du degré de complexité des ILEIS, nous a permis de distinguer au sein des ILEIS différents degrés sémiotiques pourvus de signification autonome ou non :

a) les traits saillants permettant de caractériser l'objet, l'action, etc. représentés et ne possédant donc aucune signification propre en dehors de la représentation de laquelle elle participe ;

b) l'iconème qui possède une autonomie et peut se comporter comme un motif et entrer en composition avec d'autres au sein d'une ILEIS ou alors constituer à lui seul une ILEIS ;

c) nous suggérions de désigner l'ILEIS comme un iconogramme par analogie avec le pictogramme. Ce terme éviterait toute confusion avec le terme d'icone déjà largement polysémique puisqu'il est utilisé déjà dans la typologie peircéenne, mais aussi par les développeurs et les ergonomes. Pourtant la force du langage ordinaire semblerait opposée à cet usage, le terme d'icone s'étant progressivement imposé.

4. Cette délimitation, qui correspond globalement aux trois niveaux classiques reconnus par la sémiotique visuelle (sous-entité, entité, surentité) permet de définir trois niveaux d'organisation et de structuration syntaxiques des ILEIS :

a) l'intra-iconogrammique (plus simplement, intra-iconique) qui porte sur l'organisation des traits saillants au sein d'une ILEIS ;

b) l'intericonèmique qui porte sur l'organisation des motifs, des iconèmes, au sein de l'ILEIS. C'est à ce niveau que nous avons pu définir les différentes formes d'organisation syntaxique (superposition, apposition, inclusion, condensation, etc.). Cependant du point de vue topologique, seule une description statistique permettrait de mettre en évidence certaines régularités et de les mettre en rapport avec la structure linguistique des descriptions verbales qui accompagnent les ILEIS ;

c) l'intericonogrammique qui porte sur 1'organisation des icones au sein d'une série.

5. Les formes d'organisation entre les différents iconèmes peuvent présenter un caractère analogique ou alors conventionnel. Autrement dit, l'analogique et le conventionnel qui permettent de distinguer la nature sémiotique des ILEIS et/ou de leur compo- 
santes permettent aussi d'établir, d'identifier différentes modalités d'organisation syntaxique entre les iconèmes d'une même ILEIS.

De façon plus générale, nous avions supposé l'existence d'une différence entre iconotypes et ILEIS. Il est évident que, contrairement à l'iconotype, l'ILEIS constitue une représentation conventionnelle et généralisée puisque c'est là l'essence même de l'ILEIS. L'intérêt serait alors de voir quelles sont les modifications qu'implique ce double processus de conventionalisation et de généralisation. Rappelons que dans la perspective généalogique adoptée par Darras, c'est ce processus qui mène de l'iconotype au pictogramme. L'iconotype implique la non segmentation des motifs et le respect absolu de leur identité. En conséquence, Darras observe et décrit les stratégies graphiques qui conduisent à l'évitement des procédés de composition comme la superposition, l'inclusion, etc. au profit du voisinage et de la juxtaposition.

Dans le cas des ILEIS et sans doute plus généralement dans celui des pictogrammes, la disposition semble obéir à une logique toute différente. C'est donc l'intégration des différents motifs en une représentation globale qui semble prévaloir. Le procédé de composition par condensation - intégration métaphorique - est certainement celui qui réalise le plus parfaitement cet idéal d'intégration au modèle cognitif. Mais d'autres soulignent cette tendance. D'abord le nombre d'icones agencées selon les lois de la superposition semble contredire la tendance au maintien de l'identité des motifs observée dans les iconotypes. Deuxièmement, l'existence de cas d'inclusion - rares il est vrai - paraissent constituer une étape vers la condensation et l'intégration métaphorique. Enfin, l'existence d'ILEIS sérielles indiquent l'intégration de plusieurs catégories subordonnées à une catégorie de base, comme c'est le cas pour les icones de mise en page (justification, centrage, drapeau à gauche ou à droite). Les différentes barres d'outils pourraient d'ailleurs constituer une catégorie surordonnée.

Enfin, ultime avatar de la standardisation, les ILEIS métaphoriques semblent disparaître progressivement. Si elles semblaient plus fréquentes dans l'univers $M a c$, aujourd'hui leur nombre semble décroître quel que soit l'environnement de travail. Les exemples de notre corpus sont d'ailleurs assez anciens. Mais ce point comme bien d'autres demanderait à être vérifié aujourd'hui. 
Certes nous n'avons pas répondu à toutes les questions. Mais celles que nous laissons en suspens constituent des pistes de recherches que nous croyons prometteuses. 